\title{
The Measurability of Complex-Valued Functional Sequences
}

\author{
Keiko Narita \\ Hirosaki-city \\ Aomori, Japan
}

\author{
Noboru Endou \\ Gifu National College of Technology \\ Japan \\ Yasunari Shidama \\ Shinshu University \\ Nagano, Japan
}

Summary. In this article, we formalized the measurability of complexvalued functional sequences. First, we proved the measurability of the limits of real-valued functional sequences. Next, we defined complex-valued functional sequences dividing real part into imaginary part. Then using the former theorems, we proved the measurability of each part. Lastly, we proved the measurability of the limits of complex-valued functional sequences. We also showed several properties of complex-valued measurable functions. In addition, we proved properties of complex-valued simple functions.

MML identifier: MESFUN7C, version: $\underline{7.11 .014 .117 .1046}$

The articles [12], [26], [2], [8], [1], [21], [27], [9], [11], [3], [18], [10], [22], [4], [5], [17], [23], [20], [28], [6], [7], [16], [14], [24], [19], [25], [15], and [13] provide the notation and terminology for this paper.

\section{Real-Valued Functional Sequences}

For simplicity, we adopt the following rules: $X$ is a non empty set, $Y$ is a set, $S$ is a $\sigma$-field of subsets of $X, M$ is a $\sigma$-measure on $S, f, g$ are partial functions from $X$ to $\mathbb{C}, r$ is a real number, $k$ is a real number, and $E$ is an element of $S$.

Let $X$ be a non empty set and let $f$ be a sequence of partial functions from $X$ into $\mathbb{R}$. The functor $\overline{\mathbb{R}}(f)$ yields a sequence of partial functions from $X$ into $\overline{\mathbb{R}}$ and is defined by: 
(Def. 1) $\overline{\mathbb{R}}(f)=f$.

Next we state the proposition

(1) Let $X$ be a non empty set, $f$ be a sequence of partial functions from $X$ into $\mathbb{R}$, and $x$ be an element of $X$. Then $f \# x=\overline{\mathbb{R}}(f) \# x$.

Let $X$ be a non empty set and let $f$ be a function from $X$ into $\mathbb{R}$. Observe that $\overline{\mathbb{R}}(f)$ is total.

Let $X$ be a non empty set and let $f$ be a sequence of partial functions from $X$ into $\mathbb{R}$. The functor inf $f$ yielding a partial function from $X$ to $\overline{\mathbb{R}}$ is defined by:

(Def. 2) $\inf f=\inf \overline{\mathbb{R}}(f)$.

One can prove the following proposition

(2) Let $X$ be a non empty set, $f$ be a sequence of partial functions from $X$ into $\mathbb{R}$, and $x$ be an element of $X$. If $x \in \operatorname{dominf} f$, then $(\inf f)(x)=$ inf $\operatorname{rng} \overline{\mathbb{R}}(f \# x)$.

Let $X$ be a non empty set and let $f$ be a sequence of partial functions from $X$ into $\mathbb{R}$. The functor sup $f$ yielding a partial function from $X$ to $\overline{\mathbb{R}}$ is defined by:

(Def. 3) $\sup f=\sup \overline{\mathbb{R}}(f)$.

Next we state the proposition

(3) Let $X$ be a non empty set, $f$ be a sequence of partial functions from $X$ into $\mathbb{R}$, and $x$ be an element of $X$. If $x \in \operatorname{dom} \sup f$, then $(\sup f)(x)=$ $\sup \operatorname{rng} \overline{\mathbb{R}}(f \# x)$.

Let $X$ be a non empty set and let $f$ be a sequence of partial functions from $X$ into $\mathbb{R}$. The inferior real sequence of $f$ yields a sequence of partial functions from $X$ into $\overline{\mathbb{R}}$ with the same dom and is defined by:

(Def. 4) The inferior real sequence of $f=$ the inferior real sequence of $\overline{\mathbb{R}}(f)$.

Next we state the proposition

(4) Let $X$ be a non empty set, $f$ be a sequence of partial functions from $X$ into $\mathbb{R}$, and $n$ be a natural number. Then

(i) $\operatorname{dom}($ the inferior real sequence of $f)(n)=\operatorname{dom} f(0)$, and

(ii) for every element $x$ of $X$ such that $x \in$ dom (the inferior real sequence of $f)(n)$ holds (the inferior real sequence of $f)(n)(x)=($ the inferior real sequence of $\overline{\mathbb{R}}(f \# x))(n)$.

Let $X$ be a non empty set and let $f$ be a sequence of partial functions from $X$ into $\mathbb{R}$. The superior real sequence of $f$ yields a sequence of partial functions from $X$ into $\overline{\mathbb{R}}$ with the same dom and is defined by:

(Def. 5) The superior real sequence of $f=$ the superior real sequence of $\overline{\mathbb{R}}(f)$.

We now state two propositions: 
(5) Let $X$ be a non empty set, $f$ be a sequence of partial functions from $X$ into $\mathbb{R}$, and $n$ be a natural number. Then

(i) $\operatorname{dom}($ the superior real sequence of $f)(n)=\operatorname{dom} f(0)$, and

(ii) for every element $x$ of $X$ such that $x \in \operatorname{dom}$ (the superior real sequence of $f)(n)$ holds (the superior real sequence of $f)(n)(x)=($ the superior real sequence of $\overline{\mathbb{R}}(f \# x))(n)$.

(6) Let $f$ be a sequence of partial functions from $X$ into $\mathbb{R}$ and $x$ be an element of $X$. Suppose $x \in \operatorname{dom} f(0)$. Then (the inferior real sequence of $f) \# x=$ the inferior real sequence of $\overline{\mathbb{R}}(f \# x)$.

Let $X$ be a non empty set and let $f$ be a sequence of partial functions from $X$ into $\mathbb{R}$ with the same dom. Observe that $\overline{\mathbb{R}}(f)$ has the same dom.

We now state several propositions:

(7) Let $X$ be a non empty set, $f$ be a sequence of partial functions from $X$ into $\mathbb{R}$ with the same dom, $S$ be a $\sigma$-field of subsets of $X, E$ be an element of $S$, and $n$ be a natural number. If $f(n)$ is measurable on $E$, then $(\overline{\mathbb{R}}(f))(n)$ is measurable on $E$.

(8) Let $X$ be a non empty set, $f$ be a sequence of partial functions from $X$ into $\mathbb{R}$, and $n$ be an element of $\mathbb{N}$. Then $\overline{\mathbb{R}}(f) \uparrow n=\overline{\mathbb{R}}(f \uparrow n)$.

(9) Let $f$ be a sequence of partial functions from $X$ into $\mathbb{R}$ with the same dom and $n$ be an element of $\mathbb{N}$. Then (the inferior real sequence of $f)(n)=$ $\inf (f \uparrow n)$.

(10) Let $f$ be a sequence of partial functions from $X$ into $\mathbb{R}$ with the same dom and $n$ be an element of $\mathbb{N}$. Then (the superior real sequence of $f)(n)=$ $\sup (f \uparrow n)$.

(11) Let $f$ be a sequence of partial functions from $X$ into $\mathbb{R}$ and $x$ be an element of $X$. Suppose $x \in \operatorname{dom} f(0)$. Then (the superior real sequence of $f) \# x=$ the superior real sequence of $\overline{\mathbb{R}}(f \# x)$.

Let $X$ be a non empty set and let $f$ be a sequence of partial functions from $X$ into $\mathbb{R}$. The functor liminf $f$ yields a partial function from $X$ to $\overline{\mathbb{R}}$ and is defined as follows:

(Def. 6) $\liminf f=\liminf \overline{\mathbb{R}}(f)$.

Next we state the proposition

(12) Let $X$ be a non empty set, $f$ be a sequence of partial functions from $X$ into $\mathbb{R}$, and $x$ be an element of $X$. If $x \in \operatorname{dom} \liminf f$, then $(\liminf f)(x)=$ $\liminf \overline{\mathbb{R}}(f \# x)$.

Let $X$ be a non empty set and let $f$ be a sequence of partial functions from $X$ into $\mathbb{R}$. The functor $\limsup f$ yields a partial function from $X$ to $\overline{\mathbb{R}}$ and is defined by:

(Def. 7) $\lim \sup f=\lim \sup \overline{\mathbb{R}}(f)$.

One can prove the following proposition 
(13) Let $X$ be a non empty set, $f$ be a sequence of partial functions from $X$ into $\mathbb{R}$, and $x$ be an element of $X$. If $x \in \operatorname{dom} \limsup f$, then $(\lim \sup f)(x)=\lim \sup \overline{\mathbb{R}}(f \# x)$.

Let $X$ be a non empty set and let $f$ be a sequence of partial functions from $X$ into $\mathbb{R}$. The functor $\lim f$ yielding a partial function from $X$ to $\overline{\mathbb{R}}$ is defined by:

(Def. 8) $\lim f=\lim \overline{\mathbb{R}}(f)$.

Next we state several propositions:

(14) Let $X$ be a non empty set, $f$ be a sequence of partial functions from $X$ into $\mathbb{R}$, and $x$ be an element of $X$. If $x \in \operatorname{dom} \lim f$, then $(\lim f)(x)=$ $\lim \overline{\mathbb{R}}(f \# x)$.

(15) Let $f$ be a sequence of partial functions from $X$ into $\mathbb{R}$ and $x$ be an element of $X$. If $x \in \operatorname{dom} \lim f$ and $f \# x$ is convergent, then $(\lim f)(x)=$ $(\limsup f)(x)$ and $(\lim f)(x)=(\liminf f)(x)$.

(16) Let $f$ be a sequence of partial functions from $X$ into $\mathbb{R}$ with the same dom, $F$ be a sequence of subsets of $S$, and $r$ be a real number. Suppose that for every natural number $n$ holds $F(n)=\operatorname{dom} f(0) \cap \operatorname{GT}$-dom $(f(n), r)$. Then $\bigcup \operatorname{rng} F=\operatorname{dom} f(0) \cap \mathrm{GT}-\operatorname{dom}(\sup f, r)$.

(17) Let $f$ be a sequence of partial functions from $X$ into $\mathbb{R}$ with the same dom, $F$ be a sequence of subsets of $S$, and $r$ be a real number. Suppose that for every natural number $n$ holds $F(n)=\operatorname{dom} f(0) \cap$ GTE-dom $(f(n), r)$. Then $\bigcap \operatorname{rng} F=\operatorname{dom} f(0) \cap$ GTE-dom(inf $f, r)$.

(18) Let $f$ be a sequence of partial functions from $X$ into $\mathbb{R}$ with the same dom and $E$ be an element of $S$. Suppose $\operatorname{dom} f(0)=E$ and for every natural number $n$ holds $f(n)$ is measurable on $E$. Then $\lim \sup f$ is measurable on E.

(19) Let $f$ be a sequence of partial functions from $X$ into $\mathbb{R}$ with the same dom and $E$ be an element of $S$. Suppose $\operatorname{dom} f(0)=E$ and for every natural number $n$ holds $f(n)$ is measurable on $E$. Then $\liminf f$ is measurable on E.

(20) Let $f$ be a sequence of partial functions from $X$ into $\mathbb{R}$ and $x$ be an element of $X$. Suppose $x \in \operatorname{dom} f(0)$ and $f \# x$ is convergent. Then (the superior real sequence of $f$ ) $\# x$ is lower bounded.

(21) Let $f$ be a sequence of partial functions from $X$ into $\mathbb{R}$ with the same dom and $E$ be an element of $S$. Suppose that

(i) $\operatorname{dom} f(0)=E$,

(ii) for every natural number $n$ holds $f(n)$ is measurable on $E$, and

(iii) for every element $x$ of $X$ such that $x \in E$ holds $f \# x$ is convergent. Then $\lim f$ is measurable on $E$.

(22) Let $f$ be a sequence of partial functions from $X$ into $\mathbb{R}$ with the same 
dom, $g$ be a partial function from $X$ to $\overline{\mathbb{R}}$, and $E$ be an element of $S$. Suppose that

(i) $\operatorname{dom} f(0)=E$,

(ii) for every natural number $n$ holds $f(n)$ is measurable on $E$,

(iii) $\operatorname{dom} g=E$, and

(iv) for every element $x$ of $X$ such that $x \in E$ holds $f \# x$ is convergent and $g(x)=\lim (f \# x)$.

Then $g$ is measurable on $E$.

\section{The Measurability of Complex-Valued Functional Sequences}

Let $X$ be a non empty set, let $H$ be a sequence of partial functions from $X$ into $\mathbb{C}$, and let $x$ be an element of $X$. The functor $H \# x$ yields a complex sequence and is defined by:

(Def. 9) For every natural number $n$ holds $(H \# x)(n)=H(n)(x)$.

Let $X$ be a non empty set and let $f$ be a sequence of partial functions from $X$ into $\mathbb{C}$. The functor $\lim f$ yields a partial function from $X$ to $\mathbb{C}$ and is defined as follows:

(Def. 10) $\operatorname{dom} \lim f=\operatorname{dom} f(0)$ and for every element $x$ of $X$ such that $x \in$ $\operatorname{dom} \lim f$ holds $(\lim f)(x)=\lim (f \# x)$.

Let $X$ be a non empty set and let $f$ be a sequence of partial functions from $X$ into $\mathbb{C}$. The functor $\Re(f)$ yields a sequence of partial functions from $X$ into $\mathbb{R}$ and is defined by the condition (Def. 11).

(Def. 11) Let $n$ be a natural number. Then $\operatorname{dom} \Re(f)(n)=\operatorname{dom} f(n)$ and for every element $x$ of $X$ such that $x \in \operatorname{dom} \Re(f)(n)$ holds $\Re(f)(n)(x)=$ $\Re(f \# x)(n)$.

Let $X$ be a non empty set and let $f$ be a sequence of partial functions from $X$ into $\mathbb{C}$ with the same dom. Then $\Re(f)$ is a sequence of partial functions from $X$ into $\mathbb{R}$ with the same dom.

Let $X$ be a non empty set and let $f$ be a sequence of partial functions from $X$ into $\mathbb{C}$. The functor $\Im(f)$ yields a sequence of partial functions from $X$ into $\mathbb{R}$ and is defined by the condition (Def. 12).

(Def. 12) Let $n$ be a natural number. Then $\operatorname{dom} \Im(f)(n)=\operatorname{dom} f(n)$ and for every element $x$ of $X$ such that $x \in \operatorname{dom} \Im(f)(n)$ holds $\Im(f)(n)(x)=$ $\Im(f \# x)(n)$.

Let $X$ be a non empty set and let $f$ be a sequence of partial functions from $X$ into $\mathbb{C}$ with the same dom. Then $\Im(f)$ is a sequence of partial functions from $X$ into $\mathbb{R}$ with the same dom.

Next we state several propositions: 
(23) Let $f$ be a sequence of partial functions from $X$ into $\mathbb{C}$ with the same dom and $x$ be an element of $X$. If $x \in \operatorname{dom} f(0)$, then $\Re(f) \# x=\Re(f \# x)$ and $\Im(f) \# x=\Im(f \# x)$.

(24) Let $f$ be a sequence of partial functions from $X$ into $\mathbb{C}$ and $n$ be a natural number. Then $\Re(f)(n)=\Re(f(n))$ and $\Im(f)(n)=\Im(f(n))$.

(25) Let $f$ be a sequence of partial functions from $X$ into $\mathbb{C}$ with the same dom. Suppose that for every element $x$ of $X$ such that $x \in \operatorname{dom} f(0)$ holds $f \# x$ is convergent. Then $\lim \Re(f)=\Re(\lim f)$ and $\lim \Im(f)=\Im(\lim f)$.

(26) Let $f$ be a sequence of partial functions from $X$ into $\mathbb{C}$ with the same dom and $E$ be an element of $S$. Suppose that

(i) $\operatorname{dom} f(0)=E$,

(ii) for every natural number $n$ holds $f(n)$ is measurable on $E$, and

(iii) for every element $x$ of $X$ such that $x \in E$ holds $f \# x$ is convergent. Then $\lim f$ is measurable on $E$.

(27) Let $f$ be a sequence of partial functions from $X$ into $\mathbb{C}$ with the same dom, $g$ be a partial function from $X$ to $\mathbb{C}$, and $E$ be an element of $S$. Suppose that

(i) $\operatorname{dom} f(0)=E$,

(ii) for every natural number $n$ holds $f(n)$ is measurable on $E$,

(iii) $\operatorname{dom} g=E$, and

(iv) for every element $x$ of $X$ such that $x \in E$ holds $f \# x$ is convergent and $g(x)=\lim (f \# x)$.

Then $g$ is measurable on $E$.

\section{Selected Properties of Complex-Valued Measurable Functions}

One can prove the following propositions:

(28) $\quad(r f) \uparrow Y=r(f\lceil Y)$.

(29) If $0 \leq k$ and $E \subseteq \operatorname{dom} f$ and $f$ is measurable on $E$, then $|f|^{k}$ is measurable on $E$.

(30) For all partial functions $f, g$ from $X$ to $\mathbb{R}$ holds $\overline{\mathbb{R}}(f) \overline{\mathbb{R}}(g)=\overline{\mathbb{R}}(f g)$.

(31) Let $f, g$ be partial functions from $X$ to $\mathbb{R}$. Suppose $\operatorname{dom} f \cap \operatorname{dom} g=E$ and $f$ is measurable on $E$ and $g$ is measurable on $E$. Then $f g$ is measurable on $E$.

(32) $\Re(f g)=\Re(f) \Re(g)-\Im(f) \Im(g)$ and $\Im(f g)=\Im(f) \Re(g)+\Re(f) \Im(g)$.

(33) If $\operatorname{dom} f \cap \operatorname{dom} g=E$ and $f$ is measurable on $E$ and $g$ is measurable on $E$, then $f g$ is measurable on $E$.

(34) Let $f, g$ be partial functions from $X$ to $\mathbb{R}$. Suppose that

(i) there exists an element $E$ of $S$ such that $E=\operatorname{dom} f$ and $E=\operatorname{dom} g$ and $f$ is measurable on $E$ and $g$ is measurable on $E$, 
(ii) $f$ is non-negative,

(iii) $g$ is non-negative, and

(iv) for every element $x$ of $X$ such that $x \in \operatorname{dom} g$ holds $g(x) \leq f(x)$. Then $\int g \mathrm{~d} M \leq \int f \mathrm{~d} M$.

(35) Let $X$ be a non empty set, $S$ be a $\sigma$-field of subsets of $X, M$ be a $\sigma$-measure on $S$, and $f$ be a partial function from $X$ to $\mathbb{C}$. Suppose $f$ is integrable on $M$. Then there exists an element $A$ of $S$ such that $A=\operatorname{dom} f$ and $f$ is measurable on $A$ and $|f|$ is integrable on $M$.

(36) Suppose $f$ is integrable on $M$. Then there exists a function $F$ from $\mathbb{N}$ into $S$ such that

(i) for every natural number $n$ holds $F(n)=\operatorname{dom} f \cap \operatorname{GTE}-\operatorname{dom}\left(|f|, \overline{\mathbb{R}}\left(\frac{1}{n+1}\right)\right)$,

(ii) $\operatorname{dom} f \backslash \mathrm{EQ}$-dom $(|f|, 0)=\bigcup \operatorname{rng} F$, and

(iii) for every natural number $n$ holds $F(n) \in S$ and $M(F(n))<+\infty$.

In the sequel $x, A$ denote sets.

Next we state several propositions:

(37) $|f| \uparrow A=|f \uparrow A|$.

(38) $\operatorname{dom}(|f|+|g|)=\operatorname{dom} f \cap \operatorname{dom} g$ and $\operatorname{dom}|f+g| \subseteq \operatorname{dom}|f|$.

(39) $|f| \uparrow \operatorname{dom}|f+g|+|g| \uparrow \operatorname{dom}|f+g|=(|f|+|g|)\lceil\operatorname{dom}|f+g|$.

(40) If $x \in \operatorname{dom}|f+g|$, then $|f+g|(x) \leq(|f|+|g|)(x)$.

(41) Let $f, g$ be partial functions from $X$ to $\mathbb{R}$. If for every set $x$ such that $x \in \operatorname{dom} f$ holds $f(x) \leq g(x)$, then $g-f$ is non-negative.

(42) Suppose $f$ is integrable on $M$ and $g$ is integrable on $M$. Then there exists an element $E$ of $S$ such that $E=\operatorname{dom}(f+g)$ and $\int|f+g| \mid E \mathrm{~d} M \leq$ $\int|f|\left\lceil E \mathrm{~d} M+\int|g| \mid E \mathrm{~d} M\right.$.

\section{Properties of Complex-Valued Simple Functions}

Let $X$ be a non empty set, let $S$ be a $\sigma$-field of subsets of $X$, and let $f$ be a partial function from $X$ to $\mathbb{C}$. We say that $f$ is simple function in $S$ if and only if the condition (Def. 13) is satisfied.

(Def. 13) There exists a finite sequence $F$ of separated subsets of $S$ such that

(i) $\operatorname{dom} f=\bigcup \operatorname{rng} F$, and

(ii) for every natural number $n$ and for all elements $x, y$ of $X$ such that $n \in \operatorname{dom} F$ and $x, y \in F(n)$ holds $f(x)=f(y)$.

Let $X$ be a non empty set, let $S$ be a $\sigma$-field of subsets of $X$, let $f$ be a partial function from $X$ to $\mathbb{R}$, let $F$ be a finite sequence of separated subsets of $S$, and let $a$ be a finite sequence of elements of $\mathbb{R}$. We say that $F$ and $a$ are representation of $f$ if and only if the conditions (Def. 14) are satisfied.

(Def. 14)(i) $\quad \operatorname{dom} f=\bigcup \operatorname{rng} F$,

(ii) $\operatorname{dom} F=\operatorname{dom} a$, and 
(iii) for every natural number $n$ such that $n \in \operatorname{dom} F$ and for every set $x$ such that $x \in F(n)$ holds $f(x)=a(n)$.

Let us consider $X, S, f$, let $F$ be a finite sequence of separated subsets of $S$, and let $a$ be a finite sequence of elements of $\mathbb{C}$. We say that $F$ and $a$ are representation of $f$ if and only if the conditions (Def. 15) are satisfied.

(Def. 15)(i) $\operatorname{dom} f=\bigcup \operatorname{rng} F$,

(ii) $\operatorname{dom} F=\operatorname{dom} a$, and

(iii) for every natural number $n$ such that $n \in \operatorname{dom} F$ and for every set $x$ such that $x \in F(n)$ holds $f(x)=a(n)$.

One can prove the following three propositions:

(43) $\quad f$ is simple function in $S$ if and only if $\Re(f)$ is simple function in $S$ and $\Im(f)$ is simple function in $S$.

(44) Suppose $f$ is simple function in $S$. Then there exists a finite sequence $F$ of separated subsets of $S$ and there exists a finite sequence $a$ of elements of $\mathbb{C}$ such that

(i) $\operatorname{dom} f=\bigcup \operatorname{rng} F$,

(ii) $\operatorname{dom} F=\operatorname{dom} a$, and

(iii) for every natural number $n$ such that $n \in \operatorname{dom} F$ and for every set $x$ such that $x \in F(n)$ holds $f(x)=a(n)$.

(45) $f$ is simple function in $S$ if and only if there exists a finite sequence $F$ of separated subsets of $S$ and there exists a finite sequence $a$ of elements of $\mathbb{C}$ such that $F$ and $a$ are representation of $f$.

In the sequel $c$ denotes a finite sequence of elements of $\mathbb{C}$.

One can prove the following four propositions:

(46) For every natural number $n$ such that $n \in \operatorname{dom} \Re(c)$ holds $\Re(c)(n)=$ $\Re(c(n))$.

(47) For every natural number $n$ such that $n \in \operatorname{dom} \Im(c)$ holds $\Im(c)(n)=$ $\Im(c(n))$.

(48) Let $F$ be a finite sequence of separated subsets of $S$ and $a$ be a finite sequence of elements of $\mathbb{C}$. Then $F$ and $a$ are representation of $f$ if and only if $F$ and $\Re(a)$ are representation of $\Re(f)$ and $F$ and $\Im(a)$ are representation of $\Im(f)$.

(49) $\quad f$ is simple function in $S$ if and only if there exists a finite sequence $F$ of separated subsets of $S$ and there exists a finite sequence $c$ of elements of $\mathbb{C}$ such that $\operatorname{dom} f=\bigcup \operatorname{rng} F$ and $\operatorname{dom} F=\operatorname{dom} c$ and for every natural number $n$ such that $n \in \operatorname{dom} F$ and for every set $x$ such that $x \in F(n)$ holds $\Re(f)(x)=\Re(c)(n)$ and for every natural number $n$ such that $n \in$ $\operatorname{dom} F$ and for every set $x$ such that $x \in F(n)$ holds $\Im(f)(x)=\Im(c)(n)$. 


\section{REFERENCES}

[1] Grzegorz Bancerek. The fundamental properties of natural numbers. Formalized Mathematics, 1(1):41-46, 1990.

[2] Grzegorz Bancerek. The ordinal numbers. Formalized Mathematics, 1(1):91-96, 1990.

[3] Grzegorz Bancerek and Krzysztof Hryniewiecki. Segments of natural numbers and finite sequences. Formalized Mathematics, 1(1):107-114, 1990.

[4] Józef Białas. Infimum and supremum of the set of real numbers. Measure theory. Formalized Mathematics, 2(1):163-171, 1991.

[5] Józef Białas. Series of positive real numbers. Measure theory. Formalized Mathematics, 2(1):173-183, 1991.

[6] Józef Białas. The $\sigma$-additive measure theory. Formalized Mathematics, 2(2):263-270, 1991.

[7] Józef Białas. Some properties of the intervals. Formalized Mathematics, 5(1):21-26, 1996.

[8] Czesław Byliński. The complex numbers. Formalized Mathematics, 1(3):507-513, 1990.

[9] Czesław Byliński. Functions and their basic properties. Formalized Mathematics, 1(1):55$65,1990$.

[10] Czesław Byliński. Functions from a set to a set. Formalized Mathematics, 1(1):153-164, 1990

[11] Czesław Byliński. Partial functions. Formalized Mathematics, 1(2):357-367, 1990.

[12] Czesław Byliński. Some basic properties of sets. Formalized Mathematics, 1(1):47-53, 1990.

[13] Wenpai Chang, Hiroshi Yamazaki, and Yatsuka Nakamura. The inner product and conjugate of finite sequences of complex numbers. Formalized Mathematics, 13(3):367-373, 2005.

[14] Noboru Endou and Yasunari Shidama. Integral of measurable function. Formalized Mathematics, 14(2):53-70, 2006, doi:10.2478/v10037-006-0008-x.

[15] Noboru Endou, Yasunari Shidama, and Keiko Narita. Egoroff's theorem. Formalized Mathematics, 16(1):57-63, 2008, doi:10.2478/v10037-008-0009-z.

[16] Noboru Endou, Katsumi Wasaki, and Yasunari Shidama. Definitions and basic properties of measurable functions. Formalized Mathematics, 9(3):495-500, 2001.

[17] Jarosław Kotowicz. Convergent sequences and the limit of sequences. Formalized Mathematics, 1(2):273-275, 1990.

[18] Jarosław Kotowicz and Yuji Sakai. Properties of partial functions from a domain to the set of real numbers. Formalized Mathematics, 3(2):279-288, 1992.

[19] Keiko Narita, Noboru Endou, and Yasunari Shidama. Integral of complex-valued measurable function. Formalized Mathematics, 16(4):319-324, 2008, doi:10.2478/v10037-0080039-6.

[20] Adam Naumowicz. Conjugate sequences, bounded complex sequences and convergent complex sequences. Formalized Mathematics, 6(2):265-268, 1997.

[21] Andrzej Nędzusiak. $\sigma$-fields and probability. Formalized Mathematics, 1(2):401-407, 1990.

[22] Beata Padlewska. Families of sets. Formalized Mathematics, 1(1):147-152, 1990.

[23] Beata Perkowska. Functional sequence from a domain to a domain. Formalized Mathematics, 3(1):17-21, 1992.

[24] Yasunari Shidama and Noboru Endou. Integral of real-valued measurable function. Formalized Mathematics, 14(4):143-152, 2006, doi:10.2478/v10037-006-0018-8.

[25] Yasunari Shidama and Artur Korniłowicz. Convergence and the limit of complex sequences. Series. Formalized Mathematics, 6(3):403-410, 1997.

[26] Zinaida Trybulec. Properties of subsets. Formalized Mathematics, 1(1):67-71, 1990.

[27] Edmund Woronowicz. Relations and their basic properties. Formalized Mathematics, 1(1):73-83, 1990.

[28] Hiroshi Yamazaki, Noboru Endou, Yasunari Shidama, and Hiroyuki Okazaki. Inferior limit, superior limit and convergence of sequences of extended real numbers. Formalized Mathematics, 15(4):231-236, 2007, doi:10.2478/v10037-007-0026-3.

Received December 16, 2008 